\title{
Selecting a Cost-Effective Seed for Maximizing the Social Influence Under Real-life Constraints
}

This paper was downloaded from TechRxiv (https://www.techrxiv.org).

LICENSE

CC BY-NC-SA 4.0

SUBMISSION DATE / POSTED DATE

27-04-2021 / 28-04-2021

CITATION

Biswas, Tarun Kumer (2021): Selecting a Cost-Effective Seed for Maximizing the Social Influence Under Real-life Constraints. TechRxiv. Preprint. https://doi.org/10.36227/techrxiv.14489733.v1

DOI

10.36227/techrxiv.14489733.v1 


\title{
Selecting a Cost-Effective Seed for Maximizing the Social Influence Under Real-life Constraints
}

\author{
Tarun K. Biswas, Member, IEEE, Alireza Abbasi, Member, IEEE and Ripon K. Chakrabortty, Member, \\ IEEE
}

\begin{abstract}
The Influence Maximization (IM) problem aims at maximizing the diffusion of information or adoption of products among users in a social network by identifying and activating a set of initial users. In real-life applications, it is not unrealistic to have a higher activation cost for a user with higher influence. However, the existing works on IM consider finding the most influential users as the seed set, ignoring either the activation costs of such individual nodes and the total budget or the size of the seed set, which may not be always an optimal solution, particularly from the financial and managerial perspectives, respectively. To address these issues, we propose a more realistic and generalized formulation termed as multi-constraint influence maximization (MCIM) aiming to achieve a cost-effective solution under both budgetary and cardinality constraints. Unlike the existing IM formulations, the proposed MCIM is no longer a monotone but a submodular function. As it is also proved to be an NP-hard problem, we propose a simple additive weighting (SAW) assisted differential evolution (DE) algorithm for solving the real-world problems. Experimental results on four real-world datasets show that the proposed formulation and algorithm are effective in finding a cost-effective seed set.
\end{abstract}

Index Terms-Multi-Constraint Influence Maximization, Combinatorial Optimization, Social Networks, Simple Additive Weighting, Differential Evolution.

\section{INTRODUCTION}

$\mathrm{T}$ HE use of online social networking sites is no longer limited to social interactions among friends and family members, but is ubiquitous in many business activities including product marketing, company branding and reviewing products and services [1]. In these social networking sites, a piece of information or news could become so pervasive due to the peer influence among the users [2], aka 'word-of-mouth' effect. Thus, a careful selection of a few influential users as initial product adopters or news propagators (referred as a seed set) can facilitate this diffusion process to reach out a large number of ultimate adopters or customers in a network. Identifying this seed set of nodes in a network with the maximum influence spread is popularly known as the Influence Maximization (IM) problem in social networks [3], [4].

In the classical settings of the IM problem introduced by Kempe et al. [3], the key objective is to maximize the influence spread (i.e. $\sigma(S)$ ) considering a fixed seed set size $S$ (i.e. $|S|=k$ ). Majority of the existing works have focused on this direction to find a better solution approach for this NP-hard problem [3], [5], [6]. However, these approaches assume no or similar activation/selection cost for every seed node, ignoring their practical applications, i.e. having higher selection costs for nodes with higher influence spreads. For instance, the influence of a community leader is surely

- T. K. Biswas is with School of Engineering and Information Technology at the University of New South Wales, Canberra-2600, Australia. He is also with the Department of Industrial and Production Engineering, Jashore University of Science and Technology, Jahsore-7408, Bangladesh. E-mail: t.biswas@student.adfa.edu.au

- A. Abbasi and R. K. Chakrabortty are with School of Engineering and Information Technology at the University of New South Wales, Canberra2600, Australia

Manuscript received April, 2021 higher than his/her followers in that particular community. In other words, in the competitive business environment where multiple companies are employing the same strategy, it is not unrealistic to have higher activation cost for the community leader due to his/her higher demand in the market.

In 2013, Nguyen et al. [7] first proposed Budgeted Influence Maximization (BIM) approach which sets a fixed budgetary constraint on the optimal seed set, while considering non-uniform random costs for user/nodes selection. Since BIM is also proven as an NP-hard problem, many efforts have been noticed in the literature in developing an effective algorithm [2], [8]. Banerjee et al. [8] utilized the network's community structure for distributing the budget and reducing the overlapping influence among the seed nodes. After assigning a random cost to each node, they selected the nodes with highest degrees as seed nodes from each community within the allocated budget for that particular community. Their proposed approach actually did not perform any search method for optimizing the selected seed set any further. In other words, it provides the solution by simply taking nodes with highest degrees from each significant communities within the given budget. However, they did not set any limit on the seed set size $(k)$ and can pick up the seed nodes until the budget $B$ allows to have any. Zhang et al. [1] studied the BIM problem for maximizing the total profit under multiple products adoption strategies. To solve their formulated problem, they proposed an approach named Profit Maximization with Cost Effectiveness (PMCE) that selects two candidate seed sets by modifying the greedy rules in two different directions. Specifically, one direction emphasizes on the product cost and selects the seed nodes with higher profit/cost ratio, while other direction considers the profits only and ignores the costs. With the best set 
between them, they further improved the solution quality by applying a Profit Maximization with Intelligent Selection (PMIS) algorithm that divides the entire network into some sub-networks, where each of which corresponds to one single product. After finding the seed nodes for each product, the final seed set was selected by distributing the cost among the sub-networks as like as Multiple-Choice Knapsack Problem. Overall, all the existing BIM related works exhibit two major shortcomings. Firstly, they did not set any cardinality constraint (i.e. the seed set size) which may lead to an infeasible solution from the managerial perspective. To put it differently, it may produce a solution with a very large number of seed nodes within the given budget, which may not be always manageable as it is associated with other types of overhead costs and managerial activities. Secondly, they all set arbitrary selection costs for individual nodes irrespective of their influencing or spreading powers which is also impractical [7].

Hence, this paper proposes an alternative problem formulation referred as Multi-Constraint Influence Maximization (MCIM) which aims to identify a cost-effective seed set under multiple real-life constraints, while maximizing the influence spread. To address the aforementioned first limitation of BIM, we set a cardinality constraint on seed set size in such a way that allows decision makers to choose a solution with varying length. For dealing with the second limitation of BIM as well as the classical IM problem, we assign non-uniform selection costs for individual nodes based on their influential power (rather than random allocation as in the literature). The previous research [5], [6], [9] showed that nodes with higher out-degrees are capable of producing higher spread and suitable for propagation. Hence, for this study, the selection cost of a node is set proportional to its degree centrality score.

On the contrary to the existing IM formulations, the proposed MCIM is no longer a monotone but a submodular function. To be specific, MCIM does not consider the seed nodes as the influence spread size, but the activated nodes by themselves. The key reason for the exclusion of the seed nodes from the influence spread computation is that; MCIM allows seed sets with varying sizes $|S| \leq k$, while IM considers a fixed size $|S|=k$. To exemplify, the influence spread of a set $A$ having 10 seed nodes is already higher than the set $A$ having 8 seed nodes by a number 2 if we consider the seed set itself as the influence spread. Moreover, the seed set is some sort of investment, while the influenced nodes set by them is the achievement.

We also show that it is an NP-hard problem, and therefore, propose a Simple Additive Weighting (SAW) based Differential Evolution (DE) algorithm referred as SAWDE to find a optimal or near-optimal solution within a reasonable amount of computational time. Here, SAW is used to select the potential candidates by excluding the insignificant nodes. In real-life settings, it is quite logical to select the potential candidates before optimizing the seed set. In actual practice, companies do the same as they often choose famous film stars or sport persons for advertising their products due to the higher fans/followers. Moreover, the past studies [10], [11] revealed that a great portion of nodes in a social network does not participate in the spreading process. Thus, different from the conventional degree based approaches [5], [6], Biswas et al. [12] proposed a multi-criteria based candidate selection method to reduce the drawbacks of using a single criterion. Similarly, we employ SAW to rank the nodes by considering four wellknown centrality measures (i.e., criteria), namely degree, betweenness, closeness and eigenvector [13] to select the candidate pool.

The application of SAW is not sufficient to identify the optimal solution, as it does not consider the overlapping influence. Therefore, we design an optimization framework based on DE, which is the most advanced and powerful meta-heuristic of Evolutionary class [14] for finding the optimal seed set. The relative benefits of a problemindependent meta-heuristic over a problem-specific heuristic/approximation algorithm are [15]: (i) they are easy to design and implement without knowing much about the considered problem, and (ii) due to their stochastic search, they are capable of producing diverse solutions and escaping from local optima. As there is no known single search operator that performs well for wide-range of problem instances, our proposed approach leverages the strengths of two mutation operators in a self-adaptive manner. As the performance of a meta-heuristic algorithm greatly depends on its parameters and finding their optimal values are not an easy task [14], we also adopt the self-adaptive strategies for most of them.

The novelties of this paper can be summarized as follows:

- This paper proposes an alternative formulation for IM considering multiple real-life constraints (as described in Section 2) which is a more realistic and generalized one than the existing formulations.

- We define an objective function for MCIM considering two-hop measures of the seed nodes (presented in Section 2.2) which is no longer a monotone but submodular function.

- An MCDM based meta-heuristic algorithm named SAW-DE is designed to solve the proposed MCIM problem (described in Section 3).

- A comprehensive theoretical analysis from both the problem formulation and algorithm perspectives have been carried out.

- Finally, experimental studies on four widely-used real-life datasets have been conducted to show the efficacy of the proposed formulation of MCIM as well as the SAW-DE algorithm.

The remainder of the paper is organized as follows. Section 2 presents our proposed formulation of the MCIM problem. While Section 3 describes the proposed SAW-DE algorithm for MCIM, Section 4 discusses its experimental performance. Section 5 concludes the study by providing some future research directions.

\section{Proposed formulation of the Multi- Constraint Influence Maximization (MCIM) Problem}

This section presents the proposed MCIM problem formulation which aims to identify a seed set with the maximum 
influence spread under both given budget and cardinality constraints. Table 1 illustrates our proposed MCIM along with the existing formulations of both the classical IM and BIM problems. As discussed above, the classical IM problem aims to find out a fixed-size seed set (i.e. $|S|=k$ ) ignoring the non-uniform selection cost for individual node and total budget. In contrast, Budgeted Influence Maximization (BIM) sets a budgetary constraint considering non-uniform selection costs, however, it ignores the cardinality constraint on seed set. Addressing the existing limitations, we proposed MCIM which is more generalized form of the basic IM problem. Contrary to the classical IM problem, MCIM set the cardinality constraint as $|S| \leq k$ which allows solutions of variable sizes. For instance, both solutions $\left\{u_{1}, u_{2}\right\}$ of size 2 and $\left\{u_{1}, u_{2}, u_{3}\right\}$ of size 3 are feasible if budget $B$ allows them and $k$ value is at least 3 . This flexibility will allow decision makers to choose a cost-effective optimal seed set from wide-range of options. A case study is presented later in this section to illustrate this phenomenon. Apart from this flexible cardinality constraint, MCIM sets non-uniform selection costs for individual nodes considering their influencing powers and the degree centrality of nodes to estimate their influence powers [5], [6], [9]. For this study, individual node cost is set by multiplying a positive cost factor (CF) with its normalized degree centrality score. $\mathrm{CF}$ is used just to convert the centrality scores into costs and magnify the values. Before presenting the case study, a brief introduction on the most popular diffusion models is discussed in the following subsection.

\subsection{Information Diffusion models}

There are several diffusion models to capture the influence diffusion process. However, two most widely-used models, i.e., Independent Cascade (IC) and Weighted Cascade (WC) [5], are used in this study. In both models, each node (i.e. $u$ ) in a candidate solution set $S$ will have an opportunity to influence (activate) its every outgoing neighbor $v$ by a probability $p_{u, v}$. The basic difference between IC and WC models is in the influence probability. In IC model, $p_{u, v}$ is constant for every edge, while in $\mathrm{WC}$, the influence probability $p_{u, v}$ of a node $u$ on a node $v$ depends on the number of neighbors of the node $v$ (i.e., $P_{u, v}=\frac{1}{d_{v}}$ in case of uniform edge weights, where, $d_{v}$ is the in-degree of $v$ ). Both models unfold in discrete fashion and terminate when no more activation is possible. The spread size $\sigma(S)$ of a solution set $S$ is basically the summation of the total activated nodes by $S$, resulting from the diffusion process in the network.

\subsection{Expected Influence Score (EIS)}

Traditionally, often a large number of time-consuming Monte-Carlo Simulation (MCS) are run and their average is taken to estimate the influence spread of nodes under both IC and WC models. According to the Lemma 2.1, there exists an exponential decay in the influence probability with the increase in hop-distance between the seed nodes and the nodes that could be activated or influenced. Additionally, the Three Degree Theory [16] affirms that the influence spread of a node in a network is limited within its threehop areas. Later, Gong et al. [5] further pointed out that node's global influence can be estimated by its two-hope area and proposed a metric called Local Influence Estimator (LIE) to find the estimated influence spread under two-hop areas. However, LIE is not directly applicable to MCIM, because MCIM considers solutions of varying sizes. In other words, LIE includes the seed nodes in the influence spread calculation, whereas MCIM excludes them. Thus, we adopt LIE and propose Expected Influence Score (EIS) to fit it into the MCIM. Mathematically, it can be expressed as follows:

$$
\begin{aligned}
E I S= & \sigma_{h 1}(S)+\sigma_{h 2}(S) \\
= & \left(1+\frac{1}{\left|N_{S}^{1} \backslash S\right|} \sum_{u \in N_{S}^{2} \backslash S} p \tau_{u}^{2}\right) \\
& \times \sum_{i \in N_{S}^{1} \backslash S}\left(1-(1-p)^{\tau_{i}^{1}}\right)
\end{aligned}
$$

where, $\sigma_{h 1}(S)$ and $\sigma_{h 2}(S)$ refer to the first and second-hop influence of $S, N_{S}^{1}$ and $N_{S}^{2}$ denote the one-hop and twohop neighbors of the candidate set $S$ respectively, and $p$ is the influence probability and $\tau_{i}^{1}$ and $\tau_{u}^{2}$ are the number of edges of node $i$ and $u$ within $N_{S}^{1}$ and $N_{S}^{2}$, respectively.

Lemma 2.1. Starting from an active seed node $u$ to end up with activating an inactive node $v$, there is an exponential decrease in influence probability $p_{u, v}$ with the increase in hop/path length between them.

Proof. Assume that, node 2 be the seed node in the undirected social network as shown in Fig. 1. To be influenced node 3 by the seed node 2, there are two-hop distances between them in two independent influence paths (i.e. 21-3 and 2-4-3 as shown in Fig. 1b). Although the influence probabilities for the first-hop neighbors such as $p_{2,1}, p_{2,4}$ are unconditional, the influence probabilities for the second-hop or higher-hop neighbors (i.e. nodes $\{3,6\}$ ) are conditional. For instance, $p_{1,3}$ will be effective only if $p_{2,1}$ becomes successful. Therefore, we can compute the influence probability of a seed node on its second-hop neighbors using the conditional probability theorem [17] as follows:

$$
P\left(h_{1} \cap h_{2}\right)=P\left(h_{1}\right) \times P\left(h_{2} \mid h_{1}\right)
$$

where, $P\left(h_{1}\right)$ and $P\left(h_{2}\right)$ denote the success (influence) probabilities of the first and second hop areas, respectively. $P\left(h_{1} \cap h_{2}\right)$ is the influence probability of a seed node on its two-hop neighbors and $P\left(h_{2} \mid h_{1}\right)$ is the influence probability of the seed's first-hop neighbor on its two-hop neighbor which is assumed independent under the IC model; hence it is equal to influence probability $P\left(h_{2}\right)$. Thus, for a constant influence probability $p$, the influence probability of a seed node on its $h$-hop neighbor can be expressed as $p^{h}$, leading an exponential decay of $p$ with the increase in $h$.

Definition 2.1 (Monotone function). A function $\sigma: 2^{V} \rightarrow$ $\mathbb{R}$ is called monotonically increasing (non-decreasing) if for all $A \subseteq B \subseteq V$, it gives $\sigma(A) \leq \sigma(B)$ and called monotonically decreasing (non-increasing) when it gives $\sigma(A) \geq \sigma(B)$.

Definition 2.2 (Submodular function). A function $\sigma: 2^{V} \rightarrow$ $\mathbb{R}$ is called submodular if for all $A \subseteq B \subseteq V$ and $v \in V \backslash B$, it holds the diminishing marginal return property, i.e., $\sigma(A \cup\{v\})-$ $\sigma(A) \geq \sigma(B \cup\{v\})-\sigma(B)$. 
TABLE 1: The proposed formulation of MCIM along with other existing IM formulations

\begin{tabular}{|l|l|l|}
\hline The classical IM problem [3], [5] & Budgeted IM problem [7], [8] & Proposed Multi-Constraint IM problem \\
\hline $\max , \sigma(S)$ & $\max , \sigma(S)$ & $\max , \sigma(S)$ \\
Sub. to, $|S|=k ;$ & Sub. to, $C(S) \leq B ;$ & Sub. to, $C(S) \leq B ;$ \\
& & $|S| \leq k ;$
\end{tabular}

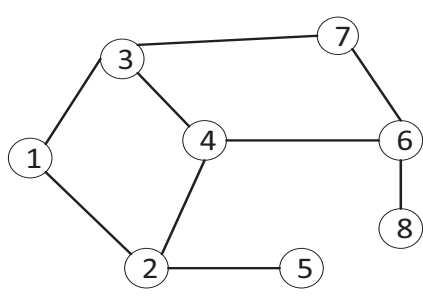

(a) social network

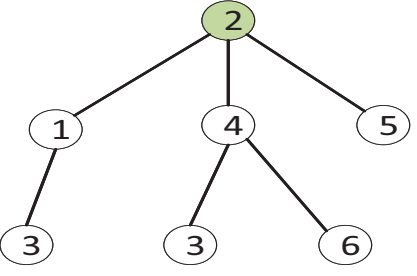

(b) influence path from node 2
Fig. 1: An example of computing EIS in 2-hop neighbor areas

An alternative definition of submodularity is that for any $A, B \subseteq$ $V$, it follows $\sigma(A \cup B)+\sigma(A \cap B) \leq \sigma(A)+\sigma(B)$.

Theorem 2.2. For any arbitrary set $S \subset V$ of a given network $G(V, E)$, the resulting $E I S(S)$ is a non-monotone function.

Proof. To compute the influence of a seed set $S$, the EIS considers the expected number of the activated nodes by $S$ in their first-hop $\left(N_{S}^{1}\right)$ and second-hop $\left(N_{S}^{2}\right)$ neighbors sets for a certain diffusion probability $p$. On the contrary to the classical IM problem, it does not consider the initial active nodes in $S$ as the influence spread. Thus, adding a node $u$ from the first-hop neighbor set of the seed set $S$ excluding the nodes that already belong to $S$ (i.e. $u \in N_{S}^{1} \backslash S$ ) to the seed set $S$ will always reduce the value of $\sigma(S \cup\{u\})$ by $1 * p$ if the node $u$ does not have any other additional neighbors. To clarify the scenario, let assume $p=1$ for the case shown in Fig. 1b. Then, the influence spread size of the seed set $\sigma(2)$ is 5 considering its first-hop $(\{1,4,5\})$ and second-hop $(\{3,6\})$ neighbor sets. Now, the inclusion of node 5 in the seed set $\{2\}$ will decrease the $\sigma(\{2\} \cup\{5\})$ by 1 as it does not add any nodes from the rest of the network to the neighbor sets of $\{2,5\}$. Since adding a node to a set $S$ can cause the EIS (i.e., $\sigma()$.$) to decrease and it violates the expression$ $\sigma(S \cup\{u\}) \geq \sigma(S)$, EIS is a non-monotone function.

Theorem 2.3. For an arbitrary instance of the cascade models (i.e. IC and WC), the resulting EIS(.) is a submodular function.

Proof. Let assume $A$ and $B$ are two sets such that $A \subset B$, and $V$ is the ground set of all nodes. If $N_{A}^{1}, N_{B}^{1}, N_{A}^{2}$, and $N_{B}^{2}$ be the sets of the first-hop and second-hop neighbors of the A and B respectively, then we have $\left(A \cup N_{A}^{1} \cup N_{A}^{2}\right) \subseteq$ $\left(B \cup N_{B}^{1} \cup N_{B}^{2}\right)$. Now, consider a node $v \notin B$, where $N_{v}^{1}$ and $N_{v}^{2}$ are the first-hop and second-hop neighbors sets of $v$ and calculate the marginal gain; i.e. $\sigma(A \cup\{v\})-\sigma(A)$. This gives us the number of elements in $\left(N_{v}^{1} \cup N_{v}^{2}\right)$ that are not already in $\left(A \cup N_{A}^{1} \cup N_{A}^{2}\right)$, which must be greater than or equal to the number of elements in $\left(N_{v}^{1} \cup N_{v}^{2}\right)$ that are not in $\left(B \cup N_{B}^{1} \cup N_{B}^{2}\right)$. More specifically, $V \backslash B$ is the maximum possible neighbors set (i.e., $\left.\left(N_{B}^{1} \cup N_{B}^{2}\right)\right)$ that decreases with adding node to the set $B$. On the other hand, the EIS takes non-negative values and it refers to the expected number of activated nodes of a seed set from its two-hop neighbors sets which is proportional to the number of elements the neighbor's sets have. Thus, it gives $\sigma(A \cup\{v\})-\sigma(A) \geq$ $\sigma(B \cup\{v\})-\sigma(B)$, which is the condition of submodulirity.

Lemma 2.4. The objective function of the classical IM problem under a cascade diffusion model is monotone only if it considers the total number of active nodes (i.e. including the seed nodes) in the network, otherwise it is non-monotone.

Proof. The proof of this Lemma is quite simple and analogous to the Theorem 2.2. Let assume, $S$ be the seed set for a social network $G(V, E)$. Therefore, $v \in V \backslash S$ are the possible candidates that could be influenced by $u \in S$. Under IC model, the influence spread size $\sigma(S)$ (excluding seed nodes) is equal to $|V \backslash S|$ when influence probability $p_{u, v}=1$ and its value will be reduced by 1 for $\sigma(S \cup\{v\})$. The same decreasing trends can be observed for other probabilistic scenarios as well. For instance, adding an immediate neighbor node $v$ of $S$ to $S$ will always reduce the value of $\sigma(S \cup\{v\})$ if the node $v$ has no other neighbors that does not belongs to the seed set. Thus, it violates the condition of monotonicity, i.e. $\sigma(S \cup\{v\}) \geq \sigma(S)$.

\subsection{A case study to assess the benefits of MCIM over the existing IM formulations}

To illustrate the efficacy of the proposed MCIM, Zachary's karate club network (containing 34 nodes and 78 edges) is used. Using brute force search as an exact method, we solve the MCIM problem and compare the results with the classical IM problem as shown in Table 2. To measure the benefit of MCIM over the classical IM, we utilize a metric called benefit/cost ratio ("b/c ratio" in Table 2). Here, the benefit is basically proportional to the EIS, where the proportionality coefficient is arbitrarily set to 100 just to magnify the differences.

As evident in Table 2, when $k=2,\{1,34\}$ is the global optimal solution set for the IM problem with benefit of 423.31. However, owing to their higher influences, the set incurs higher cost (i.e. 21.15) resulting in b/c ratio of 20.01. To compare with the IM problem, we solve the MCIM problem in two different ways. The first case offers lowering the maximum budget $B$, while keeping the fixed size seed $k$. In the second option, on the other hand, we become flexible on the seed set size $k$ up to a certain point, while keeping the fixed budget $B$. We compute the results for three different cases against each of the two cases of classical IM problem. For all the cases, the $b / c$ ratio of MCIM is found higher than the traditional IM problem, except one (row one for the flexible $k$ in Table 2 with max $k=3$ which returns the same result as IM). It is worth noticing that the b/c ratio increases with the lowering $B$ and increasing $k$ in both options respectively. However, in reality, decision makers 
can be flexible on $B$ and $k$ up to a certain limit considering the required spread level and manageability of the seed set size, respectively. More importantly, the existing problem formulations of both IM and BIM may produce an infeasible solution due to their single constraint handling capability. In this work, our proposed MCIM addresses this challenge by considering both the budgetary and cardinality constraints simultaneously, while maximizing the influence spread.

Theorem 2.5. Finding the optimal solution under MCIM problem is NP-hard.

Proof. MCIM becomes the classical IM problem, when $|S|=$ $k$ and budget $B$ is large enough to allows all possible combinations of $k$ nodes in the network as solutions (i.e. when $\forall S \in\left(\begin{array}{l}n \\ k\end{array}\right)$, the statement $C(S) \leq B$ is True). Since the classical IM problem which is already proven NP-hard [3] is a special case of MCIM problem, therefore, MCIM is also NP-hard.

\section{Proposed Algorithm for solving the MCIM PROBLEM}

Since the proposed MCIM is NP-hard, it is very difficult and often time consuming to solve it on large size realworld problems by an exact algorithm [5], [6]. Thus, we propose a simple additive weighting (SAW) assisted differential evolution (DE) algorithm referred as SAW-DE, to find a near-optimal solution by spending a reasonable amount of computation time. The proposed SAW-DE approach has two major phases: (i) candidate selection (Algorithm 1) and (ii) seed optimization (Algorithm 2). Details about these phases are described in the following subsections, while all the parameters and symbols are summarized in the nomenclature table (Table 6) at Appendix A:

\subsection{Candidate selection}

As mentioned earlier, a significant number of nodes in a network does not involve in the spreading process. Accordingly, many works [5], [6], [18] utilized the node's degree centrality score as a proxy to identify influential nodes and select them as seed candidates. However, in some cases, a node might have a high degree but its connected neighbors are not so well-connected to the other nodes in the network, making the approach ineffective. Recently, Biswas et al., [12] proposed a multi-criteria decision making (MCDM) (i.e., SAW) based approach to select the influential candidates at the preliminary phase. Following that work, we employ SAW here to rank the nodes by considering four well-known centrality measures, namely degree, betweenness, closeness and eigenvector, as the criteria for selecting influential nodes as potential seed set candidates. Note that, it is not mandatory to use these four considered centrality measures as few of them might be computationally expensive depending on the application and problem size. However, it is still better to have multiple than one as each of them has its own pros and cons.

The computational steps of SAW is presented in Algorithm 1. It goes through a linear scale transformation process as different centrality metrics might be measured in different scales and units. Thereafter, weight is assigned to individual criterion as each of them might have different impact. Generally, criteria weights are set based on expert's subjective judgment or from the previously acquired knowledge. Instead of random [12], we design a sampling based technique for computing the criteria weights as shown in Eq. 3.

$$
w_{j}=\frac{\sum_{i=1}^{q} \sigma_{j}(i)}{\sum_{j=1}^{m} \sum_{i=1}^{q} \sigma_{j}(i)} \quad \text { here, } j=1,2, \ldots, m
$$

where, $w_{j}$ denotes the weight of the $j$ th criterion, $m$ is the number of criteria used in SAW, $\sigma_{j}(i)$ is the influence spread of the $i$ node taken from the sorted influential nodes list by $j$ th criterion and $q$ is the sample size. For instance, to compute the weight for the criterion degree, take the top- $q$ nodes with highest degrees and compute the sum of the individual node's influence spread. Thereafter, the weight is computed as the ratio of the degree-based influence spread sum to the sum of the sum of the individual node's influence spreads obtained from all the criteria. It is intuitively recommended to set the $p$ value close to the $k$ value.

Once the node's influential rank is obtained by SAW, it is required to prune the insignificant nodes to move further steps. While the previous works that considered either network size $n$ [6], [18] or the seed set size $k$ for selecting the candidates set, Biswas et al., [12] developed an equation by addressing the limitations of using only one aspect of the problem. Here, we also used the same equation to determine the number of candidate nodes $(\mathrm{NoC})$ that to be taken from the obtained rank as shown in Eq. 4 .

$$
N o C=\text { roundup }\left(k+(n-k)\left(\frac{\beta k}{n}\right)^{1-\beta}\right)
$$

where, $k$ and $n$ denote to the sizes of the seed set and dataset respectively, and $\beta \in[0,1]$ is the user-defined search space control parameter. Detailed analysis on $\beta$ can be found in [12].

Algorithm 1 The key steps of SAW for nodes ranking and selecting the candidates pool

Input: A network $G$ with $n$ nodes, the considered $m$ criteria, and sample size $q$

1: Find the $m$ centrality scores for each node;

2: Build a decision matrix $C_{n, m}$ with the centrality scores $c_{i, j}$, where $i=1,2, \ldots, n$ represents the node and $j=1,2, \ldots, m$ denotes the criterion (e.g., centrality measure);

3: Transform the scale using sum method [19] as $a_{i, j}=$ $\frac{c_{i, j}}{\sum_{i=1}^{n} c_{i, j}}$ for $j=1,2, \ldots, m$;

4: Compute the criteria weights $w_{j}$ using Eq. 3 and given $q$, for $j=1,2, \ldots, m$;

5: Calculate the overall score for each node as $Q_{i}=$ $\sum_{j=1}^{m} a_{i, j} \times w_{j}$;

6: Rank the nodes based on $Q_{i}$ in descending order;

7: Compute $N o C$ using Eq. 4 and select the candidate nodes set $C_{\text {cand }}$ from the rank;

Output The influential candidate nodes list $C_{\text {cand }}$; 
TABLE 2: Results comparison between the proposed and existing formulations on karate club network under IC model

\begin{tabular}{|c|c|c|c|c|c|c|c|c|c|c|}
\hline & classical top-k I & problem & Differe & budget (B) with & ne fix & & Flexibl & $\mathrm{k}$ with the fixed budge & & \\
\hline 2 & $\{1,34\}$ & $\frac{423.31}{21.15}=20.01$ & 15 & $\{4,34\}$ & 3.17 & 21.48 & 4 & $\{3,5,17,34\}$ & 4.25 & 20.74 \\
\hline & & & 10 & $\{3,24\}$ & 2.37 & 24.60 & 5 & $\{2,5,6,28,33\}$ & 4.30 & 20.96 \\
\hline 4 & $\{1,2,33,34\}$ & $\frac{581.48}{34.62}=16.79$ & 25 & $\{1,17,25,34\}$ & 4.82 & 19.76 & 6 & $\{1,3,17,24,25,34\}$ & 5.85 & 17.20 \\
\hline & & & 20 & $\{3,13,17,34\}$ & 4.14 & 20.82 & 7 & $\{2,5,6,13,25,33,34\}$ & 5.85 & 18.25 \\
\hline
\end{tabular}

\subsection{Seed optimization}

Although SAW may provide an effective ranking of individual node, it may not be effective for finding the optimal group of influential nodes due to the overlapping influence. Therefore, in this step, we employ Differential Evolution (DE) to optimize the seed set and reduce the overlapping influence. DE is the powerful version of the evolutionary classes and has gained popularity for its simplicity and ability to fast convergence. Algorithm 2 illustrates the key steps of DE. It starts with a randomly produced initial population $X$ of size $N P$ (discussed in Section 3.2.1). In order to optimize the initial solutions, two most popular mutation strategies are employed in a self-adaptive fashion (discussed in Section 3.2.2). Thereafter, the widely-used binomial crossover [14] with a crossover factor $C_{r}$ is applied to produce an offspring population. To convert the generated infeasible solutions that violate the constraint boundaries, a repair mechanism is therefore designed, outlined in Algorithm 3. Thereafter, solutions with higher fitness values are selected for the competition in the next generation. The iterative process continues until the maximum number of generations $\left(g_{\max }\right)$ is reached. The key features of the proposed DE are described in the following subsections:

\subsubsection{Solution representation and initialization}

Since DE performs on continuous spaces and MCIM is a combinatorial optimization problem, it is required to transform the continuous solutions into discrete binary solutions. At first, a continuous value against each candidate node is generated using $\operatorname{rand}(0,1)$ function, which represents a continuous solution in the initial population. Afterwards, the Largest-Ranked-Value (LRV) [14] technique is used to convert this continuous solution into a discrete solution to select top- $k$ nodes with largest values as the seed set. An example of the solution representation system of our proposed SAW-DE algorithm is given in Fig. 2. Considering, some of the generated seed sets may not satisfy the constraints, such infeasible solutions are converted into feasible solutions using a repair mechanism as illustrated in Algorithm 3.

With this solution representation system, an initial population size $N P$ is generated on continuous space by using Latin Hyper-Cube Design (LHD) method [14]. LHD has gained popularity for its ability to produce sample points evenly from whole search space. Thereafter, it goes through the mutation, crossover and selection processes in order to find the optimal solution.

\subsubsection{Adaptive selection of mutation operators}

As discussed earlier, there is no such search operator which performs effectively for wide-range of problem instances. $\overline{\text { Algorithm } 2 \text { The key steps of DE for the seed optimization }}$

Input: A network $G$, candidate list $C_{\text {cand }}$, maximum seed set size $k$, maximum budget $B$, maximum generation $g_{\text {max }}$, population size $N P$, memory size $M$

1: Initialize the population $X$ of size $N P$ (as discussed in Section 3.2.1);

2: Convert the infeasible solutions, if any, into feasible solutions using Algorithm 3 and compute fitness values;

3: Set $g \leftarrow 0$ and $P_{o p_{1}}=P_{o p_{2}}=0.5$;

4: Calculate the values of the control parameters: $C_{r}$ and $F$ (as discussed in Section 3.2.3);

5: while $g \leq g_{\max }$ do

6: Create a mutated population using operators: $o p_{1}=D E /$ current - to $-\mathrm{rand} / 1 / \mathrm{bin} ; o p_{2}=$ $D E / \phi b e s t / 1 / b i n$;

7: Apply crossover mechanism to generate a trial vector for each target vector in the current population $X$;

8: Select solutions with higher fitness and update the current population $X$;

9: Record the successful and unsuccessful entry for each mutant and trial vector with their respective operator $o p, F$, and $C_{r}$ values in the recording memory of length $\mathrm{M}$ generations;

10: if the remainder of $(g / M)==0$ then

11: $\quad$ Update $P_{o p_{1}}$ and $P_{o p_{2}}$ values (as discussed in Section 3.2.2);

12: $\quad$ Update $C_{r}$ and $F$ values (as discussed in Section 3.2.3);

13: $\quad$ Refresh the historical memory M;

14: else

15: Continue with the current parameter values;

16: end if

17: $\quad g \leftarrow g+1$;

18: end while

19: Find the seed set $S^{*}$ with the maximum $E I S$ from $X$; Output The cost-effective seed set $S^{*}$ as solution;

\begin{tabular}{|c|c|c|c|c|c|}
\hline Candidate list & $\Rightarrow \quad 112 "$ & "257" & "67" & "891" & "9" \\
\hline \multirow[t]{2}{*}{ Continuous mode } & 0.67 & 0.24 & 0.83 & 0.11 & 0.78 \\
\hline & & \multicolumn{4}{|c|}{ I Apply LRV } \\
\hline Binary mode & $\Rightarrow$ & 0 & 1 & 0 & 1 \\
\hline
\end{tabular}

Fig. 2: An example of the solution representation system of our proposed SAW-DE algorithm, considering $k=3$ 
Algorithm 3 The pseudo-code of the repair mechanism

Input: An infeasible seed set $S^{i}$ of size $k$, the network $G$, the budget $B$

1: Set $S \leftarrow S^{i}$

2: Sort the nodes in $S$ based on individual node cost in descending order;

3: Compute the seed cost $c$ of the seed set $S$;

4: while $c>B$ do

5: Remove the last node from $S$ and update the cost $c$;

6: end while

7: Add a node with a lower cost to $S$ only if the remaining budget (i.e. $B-c$ ) allows;

Output A feasible solution $S$;

Therefore, the proposed SAW-DE algorithm employs two different mutation operators: (i) DE/current-to-rand/1/bin (Eq. 5) having good divergence property; and (ii) $D E / \phi b e s t / 1 / b i n$ (Eq. 6) having good convergence property.

- $\mathrm{DE} /$ current-to-rand/1/bin [20]

$$
u_{i, j}=\left\{\begin{array}{r}
x_{i, j}+F_{i} \cdot\left(x_{r 1, j}-x_{i, j}+x_{r 2, j}-x_{r 3, j}\right) \\
\text { if }\left(\text { rand } \leq C r_{i} \text { or } j=j_{\text {rand }}\right) \\
x_{i, j} \quad \text { otherwise }
\end{array}\right.
$$

- $\mathrm{DE} / \phi$ best $/ 1 /$ bin [14]

$$
u_{i, j}=\left\{\begin{array}{cc}
x_{\phi, j}+F_{i} \cdot\left(x_{r 1, j}-x_{r 2, j}\right) \\
\text { if }\left(\text { rand } \leq C r_{i} \text { or } j=j_{\text {rand }}\right) \\
x_{i, j} \quad \text { otherwise }
\end{array}\right.
$$

where $r_{1} \neq r_{2} \neq r_{3} \neq i$ denote the random indices, $\vec{x}_{r 1}, \vec{x}_{r 2}$ and $\vec{x}_{r 4}$ are arbitarility chosen solution from the current population, while $x_{\phi, j}$ refers to a solution taken from the best $10 \%$ of the whole population.

With the search progress, most suitable operator is emphasized based on its performance learning from past certain generations. Therefore, the success as well as failure rate of each operator is recorded in a memory of $M$ generations. The probability of applying both strategies (initially set to $P_{o p_{1}}=P_{o p_{2}}=0.5$ ) are updated frequently after completion of $M$ generations using Eq. 7 [20]:

$$
P_{o p_{i}, g}=\frac{s_{o p_{i}, g}}{\sum_{o p_{i}=1}^{2} s_{o p_{i}, g}}
$$

where $P_{o p_{i}, g}$ denotes the updated probability of $i$ th operator at generation $g, s_{o p_{i}, g}$ is the success rate of $i$ th operator during past $M$ generations, and can be computed as:

$$
\begin{array}{r}
s_{o p_{i}, g}=\frac{\sum_{j=g-M}^{g-1} n s_{o p_{i}, g}}{\sum_{j=g-M}^{g-1} n s_{o p_{i}, g}+\sum_{j=g-M}^{g-1} n f_{o p_{i}, g}}+\epsilon ; \\
(i=1,2 ; g>M)
\end{array}
$$

where $n s_{o p_{i}}$ represents the number of offspring produced by $i$ th operator that survive for the next generation, while $n f_{o p_{i}}$ denotes the failure number. To avoid the possibility of being null success rate, a trivial value $\epsilon=0.001$ is added. Once updated, the recording $M$ is deleted and refreshed.

\subsubsection{Adaptation of parameters $F$ and $C_{r}$}

The proposed SAW-DE algorithm also employs the selfadaptive strategy for both mutation parameter $F_{i}$ and crossover $C r_{i}$ (here, $i$ refers to the $i$ th solution in the current population) as they have great impacts on performance. The initial values of the parameters $C r_{i}$ and $F_{i}$ are generated using Normal and Cauchy distributions respectively, considering mean 0.5 and variance 0.1 [14]. Thereafter, a recording memory of size $M$ is also used here to track values for both parameters and updated their means as follows [14]:

$$
\begin{array}{r}
\mu_{C r}=(1-c) \mu_{C r}+c * \operatorname{mean}_{A}\left(S_{C r}\right) \\
\mu_{F}=(1-c) \mu_{F}+c * \operatorname{mean}_{L}\left(S_{F}\right)
\end{array}
$$

where $S_{C r}$ and $S_{F}$ refer to the set of all the successful values of $C_{r}$ and $F$ in memory $M, c$ takes value in range $[0,1], \operatorname{mean}_{A}($.$) denotes the arithmetic mean operation, and$ mean $_{L}$ (.) is the Lehmer mean which can be computed as $\frac{\sum_{F \in S_{F}} F^{2}}{\sum_{F \in S_{F}} F}$, where $S_{F}$ is the set of the successful entries of $F$.

\subsection{Computational complexity analysis}

In this section, we analyze the worst-case time complexity of the proposed SAW-DE approach. The time complexity of SAW is $m n$, where $m$ and $n$ denote the number of criteria and network's nodes respectively. To find the complexity of $\mathrm{DE}$, let assume, $d$ be the length of the search space (i.e. the candidates pool size, i.e. $d=N o C), g_{\max }$ is the maximum generations and $n_{p}$ is the population size at each generation. The LHD-based population initialization method has a time complexity of $O\left(n_{p} d\right)$. For each solution, both mutations as well as crossover strategies have a linear time complexity of $O(d)$, while the selection process has a constant time complexity, i.e. $O(1)$. Therefore, the time complexity considering both phases is $O(m n)+O\left(g_{\max } * n_{p}(d+d+1)\right)$. Thence, the overall time complexity of our proposed $S A W-D E$ algorithm is $O\left(g_{\max } * n_{p} * d\right)$.

\subsection{Theoretical performance of SAW-DE}

Here, we explore the theoretical performance of the proposed SAW-DE approach for solving the MCIM problem. Although SAW-DE utilizes the guided search techniques, it may behave like a R-interchange heuristic algorithm in the worst-case scenario. R-interchange heuristic proceeds with an arbitrary solution $S$ of $k$ elements, and attempts to enhance the fitness value of the solution by randomly replacing $r$ elements in $S$ with $r$ elements from $V-S$, where $r$ represents a positive integer in between $1 \leq r \leq R$. For a submodular set function, Nemhauser et al., [21] showed that $\mathrm{R}$-interchange heuristic can obtain $\frac{k}{2 k-R}$ approximation ratio. In worst-case, SAW-DE guarantees at least one element interchanged between the target vector (parent solution) and trial vector (offspring solution). Therefore, SAW-DE can provide a solution which is at least $\frac{k}{2 k-1}$ approximation within an expected run-time $O\left(g_{\max } * n_{p} * d\right)$ for the MCIM problem. 
TABLE 3: The statistical information of the four considered real-world networks

\begin{tabular}{|llllll|}
\hline Dataset & $|V|$ & $|E|$ & $\bar{d}$ & Type & Reference \\
\hline Email & 1133 & 5451 & 9.622 & Directed & {$[22]$} \\
NetScience & 1589 & 2742 & 3.45 & Multigraph & {$[22]$} \\
NetHEPT & 15233 & 58891 & 7.73 & Undirected & {$[23]$} \\
Gnutella & 62586 & 147892 & 4.73 & Directed & {$[24]$} \\
\hline
\end{tabular}

\section{EXPERIMENTAL STUDY}

In order to demonstrate the efficacy of our proposed formulation of MCIM and solution approach (i.e, SAW-DE algorithm), four popular datasets in the IM research domain are used to execute the experimental study. Table 3 presents the structural and statistical information about the datasets. Here, Email ${ }^{1}$ - an email correspondence network of a university; NetScience ${ }^{1}$ and NetHEPT ${ }^{2}$ - are two different co-authorship networks and Gnutella $^{3}$ - is a decentralized peer-to-peer file sharing network.

\subsection{Compared algorithms}

None of the existing algorithms for IM problems are directly applicable to the MCIM problem as they are all designed to handle single constraint. Thus, we first solve the MCIM problem by two baseline IM algorithms, namely CostEffective Lazy Forward (CELF) [25] and Degree Heuristics (DH) [3], without considering any budgetary constraint. Then, calculating the required maximum budget for the solutions obtained by CELF and DH, we solve the MCIM problem with our proposed SAW-DE algorithm under the same budget but considering four different settings on cardinality constraint. We refer them as $S A W-D E, S A W-$ $D E+5, S A W-D E+10$, and $S A W-D E+20$, which consider 0 , 5,10 and 20 percent additional flexibility on the seed set size, respectively, compared to the baseline algorithms. For instance, when $D H$ and CELF consider $k$ as a fixed number 20, SAW-DE, SAW-DE+5, SAW-DE+10 and SAW-DE+20 consider seed nodes up to a maximum of 20,21, 22 and 24 , respectively. In other words, the plain $S A W-D E$ basically represents the solution of the basic IM problem, whereas its other variants represent the solutions by using the core concept of the proposed MCIM problem. In reality, keeping the budget to a fixed value, the decision maker can be flexible on the cardinality constraint up to a certain point considering some other managerial constraints.

\subsection{Parameters tuning and experimental setups}

Since the performance of the DE might be affected by its parameter values, most of them are made self-adaptive except the population size $N P$, memory size $M$ and the maximum generation $g_{\max }$. For these remaining three parameters, we conduct some preliminary experiments to find the optimal values. To be specific, we distinctively conduct the experiments by setting different values for one parameter, while keeping other two as fixed. For each experimental setting, we run the $S A W-D E$ algorithm ten times to take the average value of EIS under IC model (taking $p=0.05$ and $k=30$

1. http://networkrepository.com/

2. https://arxiv.org/

3. http://snap.stanford.edu/data/ at random) on two arbitrarily selected Email and NetHEPT networks. Figs. 3a, 3b and 3c show the effects on the fitness function (i.e. EIS) for different settings of these parameter values. Based on these preliminary experiments, we set the values for the parameters $N P, M$ and $g_{\max }$ as 30,30 and 500 , respectively; the same values are used for all variants of $S A W-D E$ algorithm as well. It is worth noting that both the baseline algorithms CELF and $D H$ are parameter-less.

We also conduct the experiment for the different weight settings of the different centrality measures that considered in SAW as shown in Fig. 3d. Here, we run the algorithm on NetHEPT dataset by changing the weight $w_{j}$ for the $j$ th centrality in the range of $(0,1]$, while distributing the remaining weights to the others equally. As observed that none of the considered centrality metric able to reach the maximum fitness alone (when $w_{j}$ is equal to 1 ). All cases, it hits the peak somewhere in the middle when all four centrality measures are considered. Note that this Fig. 3d does not provide with optimal values for the criteria weights but shows the benefits of using multiple criteria. To compute criteria weights for SAW, we use the Eq. 3 by taking $q=30$ for every dataset. Based on [12], we set the $\beta$ value to 0.4 for computing $\mathrm{NoC}$.

All the programs are codded in Python and executed in a PC with Intel (R) Core ${ }^{\mathrm{TM}}$ i7-4770 CPU @ $3.40 \mathrm{GHz}$ CPU and 16GB memory. We execute all the algorithms for five discrete sets of budget and seed set constraints. For calculating required budgets, we run the baseline algorithms considering $k$ as $10,20,30,40,50$ and $C F$ as 2,000. All the simulations are carried out under the IC and WC models, while setting the influence probability $p$ to 0.05 in IC and uniform edge weights in WC model. The influence spread sizes for the seed sets obtained by various algorithms are calculated by taking average of 10,000 runs, just for the comparison purpose.

\subsection{Comparison on influence spreads}

Figs. 4 and 5 demonstrate the influence spread results for six different algorithms on four considered datasets under IC and WC models respectively. It is worth mentioning that the simulation-based CELF is the state-of-the-art IM algorithm in terms of influence spreads, while $D H$ (picks nodes with highest degrees) is known as the best for computation time [5]. It can be noticed that the plain $S A W-D E$ that represents the basic IM algorithm stands somewhere in between these two state-of-the-art IM algorithms. The key objective of our proposed MCIM algorithm reflects better on other variants of $S A W-D E$. In all cases, $S A W-D E+20$ outperforms the others, while $S A W-D E+10$ remains in the competitive position with CELF in some cases. Someone may argue that $S A W$ $D E+20$ is considering larger seed size and therefore has a better performance. However, in most real-life cases, the cost is the most important criterion in decision making process. Thus, decision makers can be flexible to some extent on the the size of the seed set, while being strict on budget. Nevertheless, there should be a limit owing to managerial and other limitations, and that should be determined by the decision maker.

It is worth noticing that the non-monotone behavior of the MCIM is vividly visible in the influence spread of $D H$ 


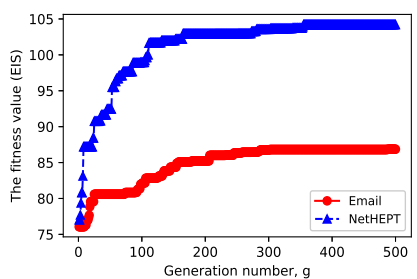

(a) The changes in EIS over $g$

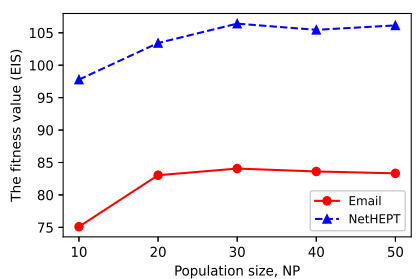

(b) The changes in EIS over NP

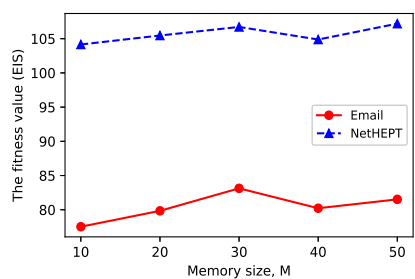

(c) The changes in EIS over $M$

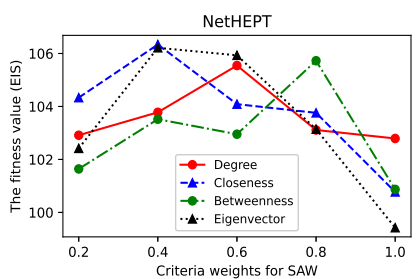

(d) The changes in EIS over $W_{j}$

Fig. 3: Here, Figs.3a, $3 \mathrm{~b}$ and $3 \mathrm{c}$ show the variations in fitness value (EIS) for different parameter values of $S A W-D E$ under IC model on two arbitrarily selected Email and NetHEPT datasets, while Fig. 3d gives the outputs of SAW-DE for different criteria weights on NetHEPT dataset under IC model.
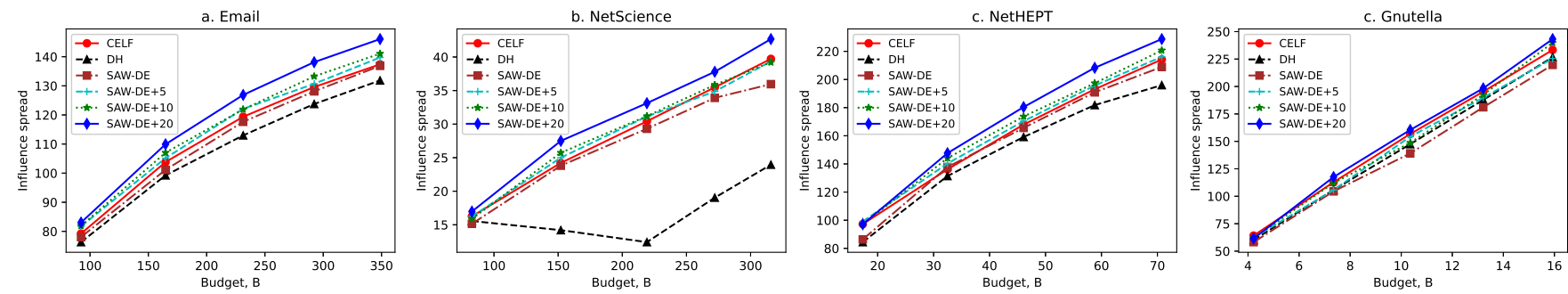

Fig. 4: The comparison on influence spread sizes of different algorithms under the IC model on four different datasets
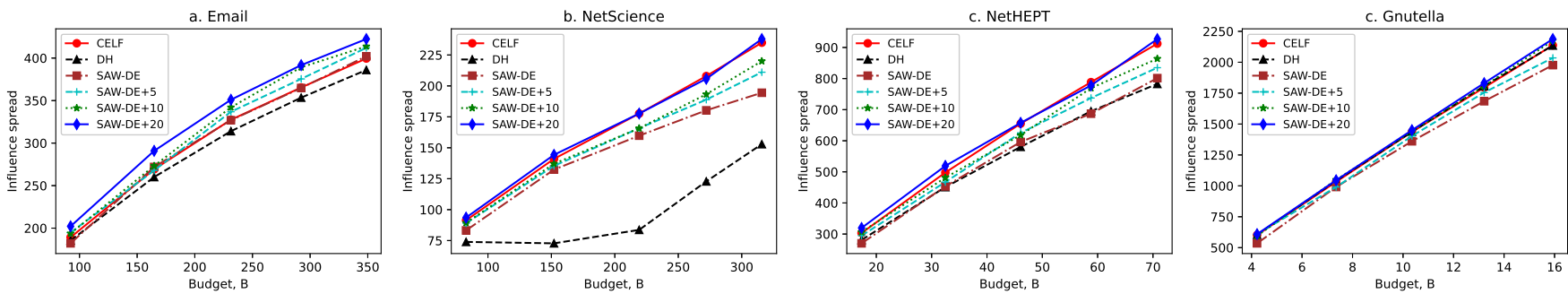

Fig. 5: The comparison on influence spread sizes of different algorithms under WC model on four different datasets

on NetScience network. As shown in Fig. 4b, the spread sizes of the $\mathrm{DH}$ algorithm decrease with the increases in the seed set sizes. More specifically, the influence spreads of the top-10 highest degree nodes set is higher than the top-30 highest degree nodes set. Since the influence spread values are fluctuating over adding members to the seed set, it proves the non-monotone behavior of the proposed MCIM problem.

\subsection{Comparison on runtime}

As the computational time of an algorithm plays an important role for solving the real-life problem, we also consider it here in our experiments. Fig. 6 presents the runtime taken by different algorithms to solve the MCIM problem. We set the y-axis in logarithmic scale to clearly see the contrast among them, while the measuring units are in second.

As illustrated in Fig. 6, the simulation based CELF took the highest computation runtime, while $D H$ stood in the first position in the rank. Note that we run the CELF algorithm only for the case of WC model on a linux platform at https://nci.org.au/, with 48 cores and partial parallelization in code. Nevertheless, it still takes the highest time. On the other hand, our proposed $S A W-D E$ and its other variants took almost similar times among themselves. Although they are not as fast as $D H$, they are much faster than CELF.

\subsection{Statistical analysis}

In this section, we conduct the non-parametric statistical analysis to assess the superiority of the compared algorithms as most of them are stochastic in nature. First, the Friedman test is employed to rank the algorithms based on the average influence spreads as shown in Table 4. As observed that $S A W-D E+20$ stands in the $1^{\text {st }}$ position in the rank with the highest overall value 5.85 , followed by the $S A W-D E+10$ and CELF respectively. In contrast, $S A W-D E$, the representative of the basic IM approach, takes the place just ahead of $\mathrm{DH}$.

TABLE 4: Rank (mean) obtained by the non-parametric Friedman test

\begin{tabular}{|l|ll|ll|ll|ll|ll|}
\hline \multirow{2}{*}{ Algorithm } & \multicolumn{2}{|l|}{ Email } & \multicolumn{2}{|c|}{ NetScience } & \multicolumn{2}{|c|}{ NetHEPT } & \multicolumn{2}{l|}{ Gnutella } & \multirow{2}{*}{ Overall mean } & \multirow{2}{*}{ Rank } \\
\cline { 2 - 9 } & IC & WC & IC & WC & IC & WC & IC & WC & & \\
\hline CELF & 3.00 & 2.80 & 3.00 & 5.20 & 2.80 & 5.00 & 4.60 & 3.20 & 3.70 & 3 \\
DH & 1.00 & 1.20 & 1.20 & 1.00 & 1.00 & 1.40 & 2.00 & 3.80 & 1.58 & 6 \\
SAW-DE & 2.00 & 2.40 & 1.80 & 2.00 & 2.20 & 1.60 & 1.00 & 1.00 & 1.75 & 5 \\
SAW-DE+5 & 4.00 & 3.80 & 4.00 & 3.00 & 4.40 & 3.20 & 3.20 & 2.60 & 3.53 & 4 \\
SAW-DE+10 & 5.00 & 4.80 & 5.00 & 4.00 & 4.80 & 4.00 & 4.60 & 4.60 & 4.60 & 2 \\
SAW-DE+20 & 6.00 & 6.00 & 6.00 & 5.80 & 5.80 & 5.80 & 5.60 & 5.80 & 5.85 & 1 \\
\hline
\end{tabular}



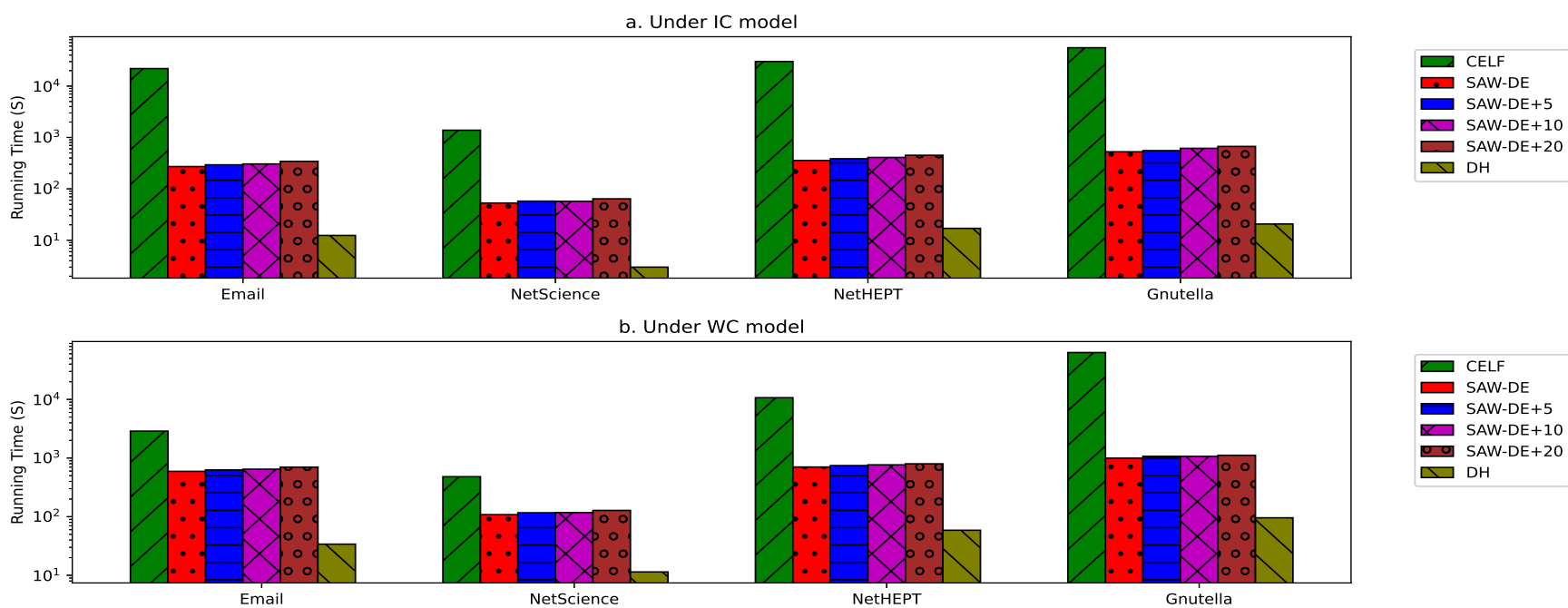

Fig. 6: The comparison on runtime (s) of different algorithms under IC and WC model on four different datasets

To detect concrete difference among the top-ranked algorithms, we also apply the Wilcoxon Signed Rank test at $5 \%$ significance level. The test result shows that there is a significance difference between $S A W-D E+20$ and $C E L F$, as the P-value is 0.001 (i.e. $\leq 0.05$ ), while $S A W-D E+10$ performs very similar to the $C E L F$, with P-value 0.096 .

In general, although $S A W-D E$ as a representative of IM algorithm is not as good as CELF, exploiting the concept of MCIM, its other variants; particularly $S A W-D E+20$ outperform the state-of-the-art IM approaches.

\subsection{Comparing the proposed MCIM with the IM and BIM formulations considering their practical feasibility}

From the experimental results and comparisons illustrated above, it is hard to understand the benefits of MCIM over BIM as they are performed basically with respect to the classical IM problem, i.e., keeping the seed size $k$ to a fixed value for the baseline algorithms. In this section, we compare our proposed MCIM with both the IM and BIM formulations to get a clear view about them, particular from the perspectives of their feasibility in the real-life applications. To do so, we consider the state-of-the-art Greedy algorithms (in terms of solution quality) as the baseline approaches which provide $\left(1-\frac{1}{e}-\epsilon\right)$ approximation [3] for the IM problem (i.e., under cardinality constraint), whereas $\left(1-\frac{1}{\sqrt{e}}\right)$ approximation [7] for the BIM problem (i.e., under cost/budgetary constraint). Here, $e$ represents the base of the natural logarithm and $\epsilon$ denotes any positive real value. To the best of our knowledge, there is no known approximation algorithm that can handle both the cardinality and cost constraints simultaneously for the submodular optimization problem.

Greedy iteratively adds node to the seed set with the maximal marginal gain (i.e., the influence spread gain for the case of IM, and the marginal gain on the influence spread/cost ratio for the BIM) and stops when the given termination condition is fulfilled (i.e., when it reaches the maximum value of: $k$ for the case of $\mathrm{IM}$, and $B$ for the BIM). Details about the Greedy algorithm for the IM (here, we used the CELF version of Greedy) and BIM problems can be found in [25] and [7], respectively. As the Greedy algorithm is computationally expensive, we consider here only two datasets Email and NetHEPT to show the contrast. Table 5 shows the results of the considered three different formulations, including our proposed MCIM. Here, the influence spread results of the SAW-DE+20 (that considers $20 \%$ additional $k$ than the IM, while using the same $B$ ), for MCIM, and CELF for IM are represented. The same settings described in subsection 4.1 are used. As the obtained seed set sizes $k$ in BIM are different from IM, we use a separate column to show them along with the influence spreads of BIM. The budget constraints $B$ for different settings of $k$ are kept fixed for all the three different formulation types.

As evident in Table 5, the influence spreads of BIM are higher than both IM and the considered case of MCIM. However, compared to the IM and MCIM, BIM selects much higher seed sets sizes that may result in higher overhead costs. For instance, managing 198 seed nodes of BIM on the NetHEPT network under IC model is not the same as managing only 10 seed nodes of IM $(198 / 10 \approx 20$ times higher). As mentioned earlier, the seed nodes are sorts of investment as they are required to be motivated to initialize the propagation process, while the set of influenced nodes by a seed set (i.e., influence spread) are the achievement. For the case of BIM, it is something like that free product samples (or any other benefits) are distributed to 198 people, just to sale 129.0 products to the other people, which do not make sense. All the cases under IC model, spread $/ k$ ratio is less than 1 for BIM, while 4.5 and 4.1 for the IM and MCIM respectively. Now, one may argue that there should not be any issues when all are utilizing the same budget. However, apart from the higher indirect overhead costs, it is more difficult to handle a larger size seed set than a smaller one.

On the other hand, the impracticality of IM arises when the available budget $B$ are not sufficient enough for selecting the top- $k$ seed nodes (as IM always aims at finding the top-k group of nodes), even though s/he can handle more than $k$ seed nodes. For instance, the required budget $B$ for selecting the top-50 seed nodes in IM on Email network is 348.7 units. On the contrary, MCIM considers 60 seed 
nodes ( $20 \%$ higher than IM) for the same budget $B$. Now, if someone wish to consider $k=60$ for IM also as like as MCIM, then it surely is not possible within this budget limit of 348.7 units.

TABLE 5: Comparing the proposed MCIM with the IM and BIM formulations considering the same budget constraints

\begin{tabular}{|c|c|c|c|c|c|c|c|c|c|c|}
\hline \multirow{3}{*}{ Dataset } & \multirow{3}{*}{$\mathrm{k}$} & \multirow{3}{*}{ B } & \multicolumn{4}{|c|}{ IC } & \multicolumn{4}{|c|}{ WC } \\
\hline & & & MCIM & IM & & BIM & MCIM & IM & & BIM \\
\hline & & & Spread & Spread & $\mathrm{k}$ & Spread & Spread & Spread & $\mathrm{k}$ & Spread \\
\hline \multirow{5}{*}{ Email } & 10 & 92.3 & 82.7 & 79.2 & 133 & 103.5 & 202.0 & 189.2 & 53 & 221.1 \\
\hline & 20 & 164.7 & 109.3 & 103.8 & 206 & 131.2 & 290.5 & 269.2 & 91 & 316.6 \\
\hline & 30 & 231.3 & 126.0 & 119.4 & 278 & 148.8 & 350.4 & 327.5 & 110 & 366.1 \\
\hline & 40 & 292.2 & 136.9 & 129.6 & 327 & 161.2 & 390.9 & 365.4 & 131 & 402.9 \\
\hline & 50 & 348.7 & 144.6 & 137.4 & 373 & 348.7 & 421.4 & 399.5 & 150 & 429.7 \\
\hline \multirow{5}{*}{ NetHEPT } & 10 & 17.3 & 97.4 & 97.2 & 198 & 129.0 & 319.4 & 303.3 & 109 & 387.8 \\
\hline & 20 & 32.5 & 148.2 & 136.2 & 309 & 186.2 & 518.6 & 497.8 & 192 & 696.7 \\
\hline & 30 & 46.1 & 181.3 & 167.9 & 383 & 225.1 & 657.0 & 655.1 & 256 & 960.7 \\
\hline & 40 & 58.8 & 209.5 & 193.1 & 481 & 256.2 & 777.0 & 788.5 & 303 & 1162.9 \\
\hline & 50 & 70.8 & 230.2 & 214.2 & 578 & 279.9 & 926.1 & 912.3 & 343 & 1352.6 \\
\hline
\end{tabular}

To sum up, none of the existing formulations provide the controlling flexibility or decision making opportunity to the users, regarding both aspects of the IM problem. To be specific, the classical IM has no control over budget $B$, while the same is true for BIM on the seed set size $k$. In contrast, our proposed MCIM can handle both constraints effectively and can trade-off by producing a more realistic and costeffective solution for IM.

\section{Conclusion}

Maximizing the diffusion of information or adoption of products among users in a (social) network through a set of initial users is known as the Influence Maximization (IM) problem. The existing formulation of the classical IM aims at identifying the most influential users as the seed set for the diffusion, while ignoring the activation costs of users and the total allocated budget for that purpose. However, in real-life applications, it is often more costly to engage users with higher influence and the budget for such projects are limited. Later, the emergence of BIM has reduced the limitations of the classical IM problem to some extent by considering the activation costs of users and total budget. Nonetheless, the BIM does not set any cardinality constraint on the seed set size that may also lead to infeasible solution, particularly from the managerial point of view. This research addresses these gaps by proposing a new realistic and generalized formulation for this optimization problem to handle multiple real-life constraints, that is referred as Multi-Constraint Influence Maximization (MCIM).

MCIM aims to achieve a cost-effective solution under both budgetary and cardinality constraints, considering particularly from the financial and managerial perspectives. Unlike the existing IM formulations, the objective function of MCIM is no longer a monotone, but a submodular function. To solve the proposed MCIM problem, we also propose an multi-criteria decision making based differential evolution (named as SAW-DE) algorithm for finding a nearoptimal solution within a reasonable amount of computational time. Experimental results on four real-life networks show that the proposed formulation of MCIM is more realistic and effective than the existing IM formulations.

The work can be expanded by considering more real-life constraints such as time, competition, and location, or by solving it as a multi-objective optimization problem. As the parameter settings may affect the results, designing a selfadaptive mechanism for SAW-DE can also be considered. In addition, investigating and developing an efficient and effective algorithm for MCIM would be another interesting future research direction.

\section{APPENDIX A}

\section{ACKNOWLEDGMENTS}

The authors would like to thank...

\section{REFERENCES}

[1] H. Zhang, H. Zhang, A. Kuhnle, and M. T. Thai, "Profit maximization for multiple products in online social networks," in IEEE INFOCOM 2016-The 35th Annual IEEE International Conference on Computer Communications. IEEE, 2016, pp. 1-9.

[2] H. T. Nguyen, M. T. Thai, and T. N. Dinh, "A billion-scale approximation algorithm for maximizing benefit in viral marketing," IEEE/ACM Transactions On Networking, vol. 25, no. 4, pp. 2419$2429,2017$.

[3] D. Kempe, J. Kleinberg, and É. Tardos, "Maximizing the spread of influence through a social network," in Proceedings of the ninth $A C M$ SIGKDD international conference on Knowledge discovery and data mining. ACM, 2003, pp. 137-146.

[4] X. Wang, Y. Zhang, W. Zhang, and X. Lin, "Efficient distance-aware influence maximization in geo-social networks," IEEE Transactions on Knowledge and Data Engineering, vol. 29, no. 3, pp. 599-612, 2016.

[5] M. Gong, J. Yan, B. Shen, L. Ma, and Q. Cai, "Influence maximization in social networks based on discrete particle swarm optimization," Information Sciences, vol. 367, pp. 600-614, 2016.

[6] S. S. Singh, K. Singh, A. Kumar, and B. Biswas, "Aco-im: maximizing influence in social networks using ant colony optimization," Soft Computing, vol. 24, no. 13, pp. 10181-10203, 2020.

[7] H. Nguyen and R. Zheng, "On budgeted influence maximization in social networks," IEEE Journal on Selected Areas in Communications, vol. 31, no. 6, pp. 1084-1094, 2013.

[8] S. Banerjee, M. Jenamani, and D. K. Pratihar, "Combim: A community-based solution approach for the budgeted influence maximization problem," Expert Systems with Applications, vol. 125, pp. 1-13, 2019.

[9] O. AskariSichani and M. Jalili, "Influence maximization of informed agents in social networks," Applied Mathematics and Computation, vol. 254, pp. 229-239, 2015.

[10] D. M. Romero, W. Galuba, S. Asur, and B. A. Huberman, "Influence and passivity in social media," in Joint European Conference on Machine Learning and Knowledge Discovery in Databases. Springer, 2011, pp. 18-33.

[11] L. Muchnik, S. Pei, L. C. Parra, S. D. Reis, J. S. Andrade Jr, S. Havlin, and H. A. Makse, "Origins of power-law degree distribution in the heterogeneity of human activity in social networks," Scientific reports, vol. 3, no. 1, pp. 1-8, 2013.

[12] T. K. Biswas, A. Abbasi, and R. K. Chakrabortty, "An mcdm integrated adaptive simulated annealing approach for influence maximization in social networks," Information Sciences, vol. 556, pp. 27-48, 2021.

[13] A. Abbasi, J. Altmann, and L. Hossain, "Identifying the effects of co-authorship networks on the performance of scholars: A correlation and regression analysis of performance measures and social network analysis measures," Journal of informetrics, vol. 5, no. 4, pp. 594-607, 2011.

[14] K. M. Sallam, R. K. Chakrabortty, and M. J. Ryan, "A twostage multi-operator differential evolution algorithm for solving resource constrained project scheduling problems," Future Generation Computer Systems, 2020.

[15] I. Wegener, "Theoretical aspects of evolutionary algorithms," in International Colloquium on Automata, Languages, and Programming. Springer, 2001, pp. 64-78.

[16] N. A. Christakis and J. H. Fowler, Connected: The surprising power of our social networks and how they shape our lives. Little, Brown Spark, 2009.

[17] A. Kolmogorov, Foundations of the theory of probability: Second English Edition. 
TABLE 6: Nomenclature

\begin{tabular}{|c|c|c|c|}
\hline Parameter & Description & Parameter & Description \\
\hline$G$ & a social graph/network & $\mathrm{Cr}$ & the crossover rate \\
\hline$n$ & the number of nodes in the graph $G$ & $F$ & the scaling parameter for mutation \\
\hline$V$ & the set of all nodes of graph $G$ & $g$ & the current generation \\
\hline$E$ & the set of all edges of graph $G$ & $g_{\max }$ & the maximum number of generation \\
\hline$S$ & the seed set to be selected & $M$ & the length of the historical memory \\
\hline$\sigma(S)$ & the fitness value/EIS of the seed set $S$ & $P_{o p_{i}, g}$ & the probability of applying $i$ th operator at generation $g$ \\
\hline$p$ & the diffusion probability & $S_{o p_{i}, g}$ & the success rate of $i$ th operator at generation $g$ \\
\hline$k$ & the number of nodes in the seed set $S$ & $i, j$ & used as indices \\
\hline$m$ & the number of criteria considered in SAW & $n s_{o p_{i}}$ & the number of successful solutions generated by $i$ th operator \\
\hline$q$ & sample size for computing criteria weights in SAW & $n f_{o p_{i}}$ & $\begin{array}{l}\text { the number of unsuccessful solutions generated by } i \text { th oper- } \\
\text { ator }\end{array}$ \\
\hline$C_{\text {cand }}$ & the candidate list of nodes & $S_{F}$ & the $F$ values utilized by successful solutions stored in $M$ \\
\hline $\begin{array}{l}\text { NoC } \\
\text { tabularnewline } \beta \\
N P\end{array}$ & $\begin{array}{c}\text { the number of nodes in the candidate list } C_{\text {cand }} \\
\text { the search space control parameter } \\
\text { the population size }\end{array}$ & $\begin{array}{l}S_{C r} \\
\mu_{F} \\
\mu_{C r}\end{array}$ & $\begin{array}{l}\text { the } C r \text { values utilized by successful solutions stored in } M \\
\text { the Lehmer mean of } S_{F} \text { that are stored in } M \\
\text { the arithmetic mean of } S_{C r} \text { that are stored in } M\end{array}$ \\
\hline
\end{tabular}

[18] M. Gong, C. Song, C. Duan, L. Ma, and B. Shen, "An efficient memetic algorithm for influence maximization in social networks," IEEE Computational Intelligence Magazine, vol. 11, no. 3, pp. 22-33, 2016.

[19] D. Stanujkic, B. Djordjevic, and M. Djordjevic, "Comparative analysis of some prominent mcdm methods: A case of ranking serbian banks," Serbian journal of management, vol. 8, no. 2, pp. 213-241, 2013.

[20] S.-Z. Zhao, P. N. Suganthan, and S. Das, "Self-adaptive differential evolution with multi-trajectory search for large-scale optimization," Soft Computing, vol. 15, no. 11, pp. 2175-2185, 2011.

[21] G. L. Nemhauser, L. A. Wolsey, and M. L. Fisher, "An analysis of approximations for maximizing submodular set functions- $\mathrm{i}$," Mathematical programming, vol. 14, no. 1, pp. 265-294, 1978.

[22] R. A. Rossi and N. K. Ahmed, "The network data repository with interactive graph analytics and visualization," in AAAI, 2015. [Online]. Available: http://networkrepository.com

[23] W. Chen, Y. Wang, and S. Yang, "Efficient influence maximization in social networks," in Proceedings of the 15th ACM SIGKDD international conference on Knowledge discovery and data mining, 2009, pp. 199-208.

[24] J. Leskovec and A. Krevl, "SNAP Datasets: Stanford large network dataset collection," http:/ /snap.stanford.edu/data, Jun. 2014.

[25] J. Leskovec, A. Krause, C. Guestrin, C. Faloutsos, J. VanBriesen, and N. Glance, "Cost-effective outbreak detection in networks," in Proceedings of the 13th ACM SIGKDD international conference on Knowledge discovery and data mining, 2007, pp. 420-429.

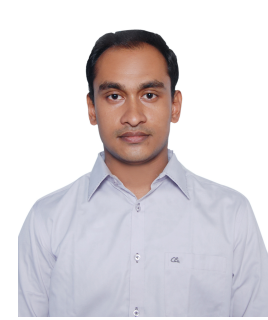

Tarun K. Biswas (Member IEEE)

is currently pursuing his $\mathrm{PhD}$ in Systems Engineering at the School of Engineering and Information Technology, University of New South Wales, Canberra, Australia. He also works as an Assistant Professor (currently on study leave) in the Department of Industrial and Production Engineering at Jashore University of Science and Technology, Jashore, Bangladesh. He completed his B.Sc. in Industrial and Production Engineering from Rajshahi University of Engineering and Technology (RUET), Bangladesh, and M.Sc. in Industrial and Production Engineering from Bangladesh University of Engineering and Technology (BUET), Bangladesh, in the years 2012 and 2018 respectively. He has more than 7 years of work and research experiences in Bangladesh. His research interest covers a wide range of topics in Operations research, Soft Computing, Optimization, Project management, Modelling and Simulation, Decision making under risk and uncertainty.

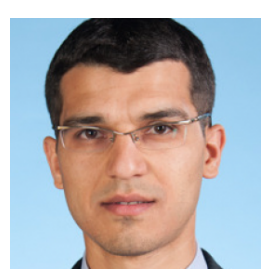

Alireza Abbasi (Member IEEE)

received his B.Sc. in Computer and Software Engineering from Isfahan University of Technology, Iran, M.Sc. in Industrial Engineering from Seoul National University, South Korea and PhD in Computer and Information Science from the University of Sydney, Australia in 2002, 2009 and 2012 respectively. $\mathrm{He}$ is one of the senior faculty members at the School of Engineering and Information Technology, University of New South Wales, Canberra, Australia. He is also the Director of Postgraduate Studies at the School of Engineering and IT at UNSW Canberra. He has published more than 68 articles/papers in the world's top tier journal and conference venues. His research aims to enhance better functioning and performance of complex socio-technical systems, which primarily lies in the emerging field of Social Computing, leveraging the capacity to collect and analyse data to reveal patterns of individual and group behaviour.

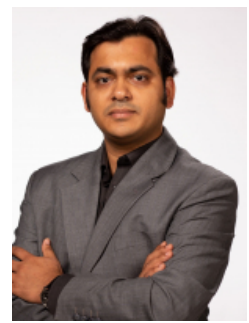

Ripon K. Chakrabortty (Member, IEEE) is the Deputy Director of the Capability Systems Centre (CSC) and lecturer on System Engineering \& Project Management at the School of Engineering and Information Technology, the University of New South Wales (UNSW Australia), Canberra. $\mathrm{He}$ is also the program coordinator for Master of Decision Analytics \& Master of Engineering Science. Dr Chakrabortty leads the optimisation and machine learning efforts in the CSC. He is currently the Group Leader of CrossDisciplinary Optimisation Under Capability Context Research Team. He obtained his PhD from the same University in 2017, while completed his MSc and BSc from Bangladesh University of Engineering \& Technology on Industrial \& Production Engineering in 2013 and 2009 respectively. He has written two book chapters and over 120 technical journal and conference papers. His research interest covers a wide range of topics in Operations Research, Project Management, Supply Chain Management, Artificial Intelligence, Cyber-physical systems and information systems management. His research program has been funded by many organisations, such as Department of Defence- Commonwealth Government, Australia. 